\title{
Exploring Neuropsychiatric Interphase in Perinatal Period - A Case Series
}

\author{
Abhishek Gupta ${ }^{1}$, Shubhangi S. Dere ${ }^{2}$, Rahul Mishra ${ }^{3}$, Rakesh Ghildiyal ${ }^{4}$ \\ ${ }^{1}$ Junior Resident, \\ ${ }^{2}$ Assistant Professor, \\ ${ }^{3}$ SeniorResident, \\ ${ }^{4}$ Professor and Head, \\ Department of Psychiatry, Mahatma Gandhi Mission Medical College, Navi Mumbai. \\ Corresponding author: Abhishek Gupta \\ Email - drabhishek096@gmail.com
}

\begin{abstract}
Perinatal period spans from conception till one-year post-partum. Pregnancy and post-partum is characterised by significant biological changes in a woman's body including changes in blood pressure, blood volume, coagulability of blood and changes in immunity causing onset or relapse of certain medical and neurological conditions, which can face diagnostic and treatment challenges during this period. We aim to present a series of 3 cases which presented with psychiatric symptoms but on further evaluation were found to have neurological diagnosis - Cavernous Venous Thrombosis [CVT], Posterior Reversible Encephalopathy Syndrome [PRES] \& Tuberculoma.
\end{abstract}

Key words: Perinatal period, CVT, PRES, tuberculoma.

(Paper received $-10^{\text {th }}$ December 2019, Peer review completed $-20^{\text {th }}$ December 2019)

(Accepted $-22^{\text {nd }}$ December 2019)

\section{INTRODUCTION}

Acute neurological diseases requiring hospitalization are relatively rare in women of childbearing age. However, during pregnancy and the postpartum period, several diseases increase in prevalence, which includes those unique to the pregnant/postpartum state including preeclampsia and delivery-associated neuropathies and others, although indirectly related to pregnancy, such as cerebral venous thrombosis, ischemic stroke, and intracerebral haemorrhage [1].

Many neurological conditions can present with initial non-specific symptoms like headache which can get mislabelled as a normal symptom of pregnancy. Neurological diseases can lead to devastating complications if not recognized early. Also, the diagnostic difficulty can arise as the pregnant woman is vulnerable in that many medications and diagnostic evaluations are avoided due to concerns of causing harm to the foetus. As neurological diseases contribute to approximately $20 \%$ of maternal deaths [2], it is critical that identification these at-risk patients is done earliest. In addition, treatment options are often limited during perinatal period considering risk of medicines to baby in utero and through breast-feeding.

Considering the overlap of neurological and psychiatric symptoms in many medical conditions like pregnancy induced hypertension, stroke, migraine, and cerebral lesions; leading to mis or underdiagnosis of serious conditions which can complicate the course and outcome of the pregnancy and post-partum it is essential to understand the interplay of the symptoms. There exists limited literature describing the neurological and psychiatric overlay during perinatal period. 


\section{CASE SERIES}

\section{Case 1}

Mrs T 30-year-old Muslim female, G2P1L1 with 8 weeks of gestation, married for 4 years got admitted in gynaecology ward for complaints of excessive vomiting in current pregnancy and severe headache for 7 days. Psychiatry reference was made as in the ward, she developed altered behaviour like irrelevant talk, getting blanked out while communicating, repeating that she can't bear headache and wants to terminate the current pregnancy. She was observed to have self-neglect and poor identification of her relatives. Such behaviours kept fluctuating with intermittent normal communication.

Pre-morbidly, patient had obsessive compulsive personality traits wherein, she had excessive concerns for cleanliness, had overvalued beliefs which she would insist family members to follow, however was functional. She did not have any psychiatric illness in past including perinatal period.

Hemogram was within normal limits except low Haemoglobin $(7.7 \mathrm{mg} / \mathrm{dl})$. No papilloedema on fundus examination.

On Mental status examination, she was poorly attentive, had perplexed affect, irrelevant talk, poor thought content, perseveration and gave approximate answers. She denied any depressive thoughts or delusions or perceptual abnormality. Her immediate memory was impaired.

MRI brain and Neurology opinion was advised in view of recent onset of altered behaviour, fluctuating course of symptoms, poor orientation and perseveration considering history of hyperemesis gravidarum. MRI Brain (Plain) and MR venography showed Venous haemorrhagic infarct in left temporal lobe. Left transverse \& sigmoid sinus thrombosis.

Figure 1: Venous haemorrhagic infarct in left temporal lobe. Left transverse \& sigmoid sinus thrombosis

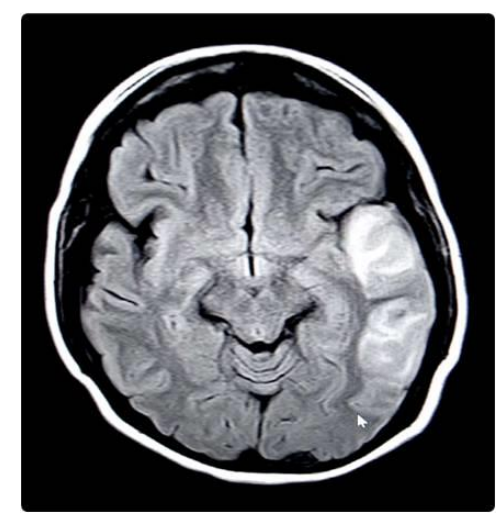

Medical termination of pregnancy was advised with consent of patient and partner. She was started on heparin derivative. Patients behavioural symptoms improved significantly with treatment. Patient was later psycho-educated to deal with her pregnancy loss.

\section{Case 2}

Mrs K, 21-year-old, G2A1 at 32 weeks of gestation (had $1^{\text {st }}$ missed abortion in $3^{\text {rd }}$ month last year), came with complaints of heaviness in right lower limb with loss of sensation for 15 minutes at presentation. On admission to obstetrics and gynaecology ward, she was observed to have high blood pressure $(180 / 110 \mathrm{~mm}$ $\mathrm{Hg}$ ) which was later stabilised with Tab. Labetalol 100mg per oral three times day. Psychiatry reference was made considering isolated limb heaviness and excessive anxiety with presence of familial stressors and absence of focal neurological deficits. Patient expressed pervasive anxiety symptoms throughout pregnancy related to her health and concerns related to childbirth. Denied any psychiatric illness in past or family.

On Mental Status Examination, she had anxious cognition with theme of well- being of baby. Patient was psycho-educated and Tab. Lorazepam 1mg was started in view of sleep disturbances and pregnancy-specific anxiety. Eventually on same day, she developed involuntary movements of right lower limb, jerky type, 
lasting for 2-3 minutes followed by weakness in lower limb for which neurology opinion advised. Her MRI brain revealed multiple areas of hyperintensity suggestive of post ictal changes. She was diagnosed with PRES (Posterior Reversible Encephalopathy Syndrome) associated with hypertension.

Figure 2: Posterior Reversible Encephalopathy Syndrome with Pregnancy Induced Hypertension

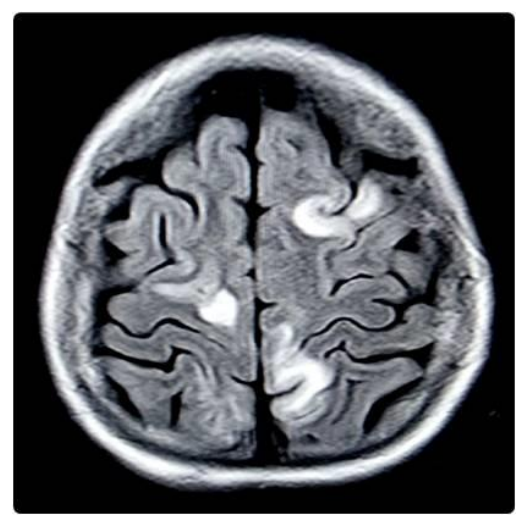

Patient was started on treatment for eclampsia protocol. Elective caesarean section was performed with postnatal observation prior to discharge in good health on $4^{\text {th }}$ day. Patient was counselled and taught relaxation exercise. Pharmacotherapy was not advised considering symptomatic improvement in her anxiety symptoms post-delivery.

\section{Case 3}

Mrs A, 25 years old Muslim female, G3P3L2 with post-partum 6 months was admitted to medicine ward for generalised weakness and poor appetite. In past history, she had completed anti-tubercular regimen 10 months back. Psychiatry reference was done for altered behaviour observed in ward which included patient having fearfulness especially in evening, shouting that she is seeing snakes on bed. She started mistaking saline tubes as snakes hanging on stand and wanted to run away from ward. On psychiatric history taking, patient did not have any psychiatric illness in past, however expressed financial stressors currently and had occasional worrying thoughts related to same since post-partum. Had disturbed sleep and appetite throughout post-partum phase and was paying less attention to self which family members thought is because of being busy with baby.

On Mental Status Examination, patient was oriented, apprehensive, helpless, hopeless, had referential thoughts, denied any delusions and had visual hallucinations. On $2^{\text {nd }}$ day, patient also developed involuntary jerky movements of both upper \& lower limbs (generalised tonic-clonic type) for which a neurology reference was taken. On hemogram, she had anaemia and leukocytosis. Neuroimaging revealed calcified tuberculoma in left occipital lobe with perilesional edema.

Figure 3: Calcified tuberculoma in left occipital lobe with perilesional edema

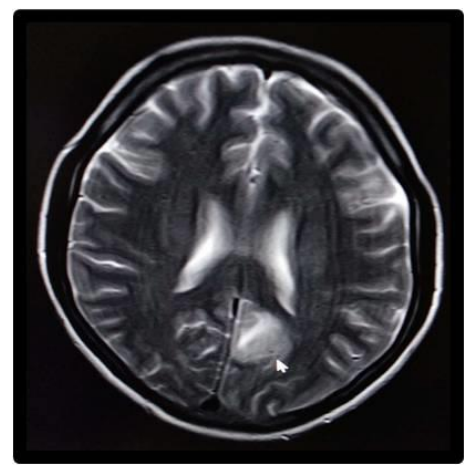


Patient was started on anti-tubercular drugs and antiepileptics. Patient and husband counselled for use of contraception as a part of preconception counselling. She was discharged in 2-3 days when she was seizurefree and behaviourally better. Tab. Sertraline was advised in view of her depressive thoughts along with psychotherapy.

\section{DISCUSSION}

Pregnancy is a well-established cause of venous thromboembolism (VTE), including intracranial venous thrombosis. Several physiological changes in coagulation system render pregnancy as prothrombotic state [3]. Diagnosis of CVT can be challenging given the variable presentation and concerns regarding neuroimaging in the pregnant patient. Venous thrombosis often presents in a subacute fashion with symptoms such as headache which are not explored further until more dramatic symptoms demand attention. Also, presence of altered behavior can cause confusion when the woman has underlying stressors and or personality factors. The diagnosis of stroke is considered in patients who present with acute onset of focal neurological changes like headache and altered mental status in the absence of an alternative etiology [4-5]. Similar case report, describes a woman with hyperemesis gravidarum, who presented with sudden onset of blurring of vision and headache who had superior sagittal sinus thrombosis [6].

PRES or reversible posterior leukoencephalopathy syndrome [RPLS] is a rare cliniconeuroradiological entity associated with a number of conditions including preeclampsia, eclampsia, severely high blood pressure, renal failure, systemic lupus erythematosus, and the assumption of immunosuppressive agents [7]. Clinical features of PRES include headache, encephalopathy, seizures, cortical visual changes, and parietooccipital white matter edema visualized on neuroimaging modalities. Even though PRES is increasingly recognized and reported in the current literature, its incidence is still obscure [8].

Considering the evolving nature of the condition and wide cerebral involvement, it may be assumed that the initial symptoms may be misunderstood as psychological symptoms as it happened in our patient. Her psycho-social stressors like previous abortion, precious pregnancy and familial stressors must have led to assume the initial symptoms to be of psychological origin. Data on neuropsychiatric overlay in PRES syndrome is sparse.

$\mathrm{TB}$ is one of the leading causes of death in women of reproductive age (15-45 years); globally it is estimated that as many as 216500 pregnant women have active TB and accounts for $15-35 \%$ of indirect maternal deaths [9]. Pregnancy and post-partum state adversely affect women's immune system that exposes them to higher risk of quiescent infections reactivation. Studies regarding pregnant women reported that the risk of $\mathrm{TB}$ infection and its related post-partum complications are twice compared to other women. In fact, one study claimed that extra-pulmonary TB disease consisted $90 \%$ of post-partum tuberculosis [10]. Psychiatric symptoms like hallucination, psychosis, altered mental status are reported in case with intracranial tuberculosis along with usual symptoms like headache, seizure [11]. The symptoms vary as per location of the tuberculoma. Visual hallucinations and palinopsia are also reported due to an occipital lobe tuberculoma [12]. Another case reports one episode of visual hallucination in a patient with tuberculous meningitis [13].

\section{CONCLUSION}

All three cases presented with initial psychological symptoms and eventually developed neurological presentations. Their psycho-social stressors needed attention and support. A team work by a gynaecologist, psychiatrist, physician and neurologist can help diagnose these rare yet serious overlapping medical conditions and help in getting better pregnancy outcome and overall well-being of mother and baby.

\section{REFERENCES}

1. Hosley CM, McCullough LD. Acute neurological issues in pregnancy and the peripartum. The Neurohospitalist 2011;1(2):104-16.

2. Berg CJ, Chang J, Callaghan WM, Whitehead SJ. Pregnancy-related mortality in the United States, 19911997. Obstetr Gynecol 2003;101(2):289-96. 
3. Khealani B, Mapari UU, Sultanali RS. Obstetric cerebral venous thrombosis. J Pak Med Assoc (JPMA) 2006;56(11):490-1.

4. Hirsch KG, Froehler MT, Huang J, Ziai WC. Occurrence of perimesencephalic subarachnoid hemorrhage during pregnancy. Neurocrit Care 2009;10(3):339-43.

5. Plewa MC, Dulebohn SC. Cavernous Sinus, Thrombosis. In Stat Pearls [Internet] 2017 Oct 6. StatPearls Publishing.

6. Cantu C, Barinagarrementeria F. Cerebral venous thrombosis associated with pregnancy and puerperium. Review of 67 cases. Stroke 1993;24(12):1880-4.

7. Poma S, Delmonte MP, Gigliuto C, Imberti R, Delmonte M, Arossa A, Iotti GA. Management of posterior reversible syndrome in preeclamptic women. Case Rep Obstetr Gynaecol 2014;2014.

8. Ural ÜM, Balik G, Şentürk Ş, Üstüner I, Çobanoğlu U, Şahin FK. Posterior reversible encephalopathy syndrome in a postpartum preeclamptic woman without seizure. Case Rep Obstetr Gynaecol 2014;2014.

9. Sobhy S, Babiker ZO, Zamora J, Khan KS, Kunst H. Maternal and perinatal mortality and morbidity associated with tuberculosis during pregnancy and the postpartum period: a systematic review and metaanalysis. BJOG 2017;124(5):727-33.

10. Alinaghian M, Salehi H, Salehi MM. A Scarce Presentation of Mycobacterium tuberculosis, Case Report. Adv Infect Dis 2018;8(1):17.

11. Werring DJ, Marsden CD. Visual hallucinations and palinopsia due to an occipital lobe tuberculoma. J Neurol Neurosurg Psychiatry 1999;66(5):684-5.

12. Chopra S, Siwatch S, Aggarwal N, Sikka P, Suri V. Pregnancy outcomes in women with tuberculosis: a 10year experience from an Indian tertiary care hospital. Trop Doctor 2017;47(2):104-9.

13. Tan JL, Nordin S, Besari AM. Rare clinical presentation of tuberculous meningitis: a case report. Malaysian J Med Sci 2017;24(5):119.

$* * * * * * * * * * * * * * * * * * * * * * * * * * * * * * * * * * *$

Acknowledgements - Nil

Conflict of Interest - Nil

Funding - Nil. 